\title{
Effect of Head Shape Variations Among Individuals on the EEG/MEG Forward and Inverse Problems
}

\author{
Nicolás von Ellenrieder, Member, IEEE, Carlos H. Muravchik, Senior Member, IEEE, Michael Wagner, \\ and Arye Nehorai, Fellow, IEEE
}

\begin{abstract}
We study the effect of the head shape variations on the EEG/magnetoencephalography (MEG) forward and inverse problems. We build a random head model such that each sample represents the head shape of a different individual and solve the forward problem assuming this random head model, using a polynomial chaos expansion. The random solution of the forward problem is then used to quantify the effect of the geometry when the inverse problem is solved with a standard head model. The results derived with this approach are valid for a continuous family of head models, rather than just for a set of cases. The random model consists of three random surfaces that define layers of different electric conductivity, and we built an example based on a set of 30 deterministic models from adults. Our results show that for a dipolar source model, the effect of the head shape variations on the EEG/MEG inverse problem due to the random head model is slightly larger than the effect of the electronic noise present in the sensors. The variations in the EEG inverse problem solutions are due to the variations in the shape of the volume conductor, while the variations in the MEG inverse problem solutions, larger than the EEG ones, are caused mainly by the variations of the absolute position of the sources in a coordinate system based on anatomical landmarks, in which the magnetometers have a fixed position.
\end{abstract}

Index Terms-EEG/magnetoencephalography (MEG) average head model, polynomial chaos expansion (PCE), sparse grids, stochastic modeling.

\section{INTRODUCTION}

$\mathbf{T}$ HE EEG/magnetoencephalography (MEG) inverse problem corresponds to estimating the parameters of sources of electromagnetic activity in the brain from measurements of the electric potential on the scalp and the magnetic field near the head. One of the factors that affects the inverse problem solution is the shape of the head model. We are interested in determining the quality of inverse problem solutions when the shape of the head is not known.

Manuscript received February 13, 2008; revised May 14, 2008 and September 18, 2008. First published October 31, 2008; current version published April 15, 2009. The work of N. von Ellenrieder and C. H. Muravchik was supported by the Universidad Nacional de La Plata (UNLP), by the Consejo Nacional de Investigaciones Científicas y Técnicas (CONICET), by the Comisión de Investigaciones Científicas de la Provincia de Buenos Aires (CICpBA), and by the Agencia Nacional de Promoción Científica y Tecnológica (ANPCyT) under Grant PICT 11-14111. Asterisk indicates corresponding author.

${ }^{*} \mathrm{~N}$. von Ellenrieder is with the Department of Electrical Engineering and the Laboratory of Industrial Electronics, Control, and Instrumentation, Faculty of Engineering, National University of La Plata, Buenos Aires 1900, Argentina (e-mail: nellen@ieee.org).

C. H. Muravchik is with the Laboratory of Industrial Electronics, Control, and Instrumentation, Faculty of Engineering, National University of La Plata, Buenos Aires 1900, Argentina.

M. Wagner is with Compumedics Neuroscan, Hamburg 20255, Germany.

A. Nehorai is with the Department of Electrical and Systems Engineering, Washington University in St. Louis, St. Louis, MO 63130 USA.

Digital Object Identifier 10.1109/TBME.2009.2008445
The effect of the head model geometry on the EEG/MEG forward and inverse problems has been considered in several studies. The difference in the EEG inverse problem solution when using spherical or realistically shaped models was studied by many authors [1]-[5]. The effect of variations in the skull thickness was studied in [6]-[8]. The effect of random variations in the head shape on the EEG forward and inverse problems was studied in [9], but the analysis was restricted to variations of a few millimeters. The localization error when solving the inverse problem with head models from several different individuals was studied in [10] and [11]. These studies analyzed the effect of the model geometry presenting the results for particular cases of head models. In this paper, we seek more general results by adopting a random head model to represent a whole family of models, and solving the EEG/MEG forward and inverse problems with it.

To study the effect of head shape variations among individuals, first we build a random head model such that each sample represents a possible shape of the head. The model is derived from a set of observed head shapes from different individuals. In this paper, we build a random head model based on $30 \mathrm{de}-$ terministic head models from adult individuals, and solve the EEG/MEG forward problem with this model. In addition, we define an average or standard head model as the expectation of the random one and use it in the solution of the EEG/MEG inverse problem.

With the random head model, the forward problem solution is also of random nature. We find the coefficients of a polynomial chaos expansion (PCE) [12], [13] of the forward problem solution. Such an expansion allows for an easy computation of the statistical moments of the solution, and hence of the variations induced in the forward problem solution by the randomness of the head model. A similar analysis could also be accomplished with Monte Carlo simulations (MCSs). However, given the high dimensionality of the problem, it would be computationally expensive. In fact, we compare the results of our method with an MCS for a reduced-size problem. The method we describe in this paper yields results similar to MCS, with a lower computational load.

To determine the effect of the random geometry on the EEG/MEG inverse problem, we assume that only the average head model is known, and compute the error in the estimation of position parameters of a dipolar source. The idea is to find the source parameters that, when used for solving the forward problem with the average head shape, minimize the difference with the forward problem solution obtained with samples of the random head model. In this way, through MCSs, we obtain a 
statistical characterization of the localization error due to the randomness of the head model.

\section{HEAD MODEL}

In this section, we propose a random model for the head shape such that each sample of the model corresponds to a head shape from a different individual. We adopt a head model with three nested layers of constant electric conductivity representing the brain, skull, and scalp tissues. The model is then defined by the three interfaces between the layers. We describe these surfaces by their radius (distance to the origin) as a function of the azimuth and elevation, with respect to a suitable coordinate system. The random head model then consists of three random surfaces $\mathcal{S}^{1}, \mathcal{S}^{2}$, and $\mathcal{S}^{3}$, describing the interfaces between tissues.

In this section, we present a procedure to obtain the random surfaces $\mathcal{S}^{q}$ as a Karhunen-Loeve series expansion truncated to a finite number $N_{c}$ of terms

$$
\mathcal{S}^{q}=S_{0}^{q}+\sum_{i=1}^{N_{c}} \chi_{i} S_{i}^{q}, \quad q=1,2,3
$$

where $S_{0}^{q}$ is a mean or standard surface and $\chi_{i}$ are uncorrelated random variables with zero mean and unit variance. Note that since the Karhunen-Loeve series expansion is assumed convergent, the surface components $S_{i}^{q}$ will tend to be smaller for higher order terms. The size of these surface components will determine the appropriate number of terms $N_{c}$.

The procedure used to build the random head model is based on the study in [10], modified to obtain an average head model and combine the individual models into an unique random one. We start from a set of observed head models corresponding to $N_{I}$ different individuals. First, we align the models; the y-direction is given by the line joining the left and right preauricular points, defining segment $\overline{a b}$. The $\mathrm{z}$-direction is normal to the plane defined by segment $\overline{a b}$ and the nasion, with positive values toward the top of the head. The origin of the coordinate system is roughly in the center of the brain, at a point $50 \mathrm{~mm}$ above the intersection between segment $\overline{a b}$ and a line normal to this segment passing through the nasion.

The observed head models used to build the random model may differ in their lower sections, e.g., if the neck was included in the model. These differences are not related to the anatomical variations we want to study. Hence, we replaced the lower part of the models, i.e., elevations under $-45^{\circ}$, by the average of the $N_{I}$ models. A linear transition of the actual head to the average shape between $-25^{\circ}$ and $-45^{\circ}$ was adopted.

Let $R_{j}^{q}, j=1, \ldots, N_{I}$, be the the interfaces between tissues of different electric conductivities of the observed head models, and let us assume that only a set of points of these surfaces is known, e.g., from a segmentation of an MRI. Next, a description is found of each brain-skull interface $R_{j}^{1}$ based on spherical harmonics

$$
R_{j}^{1}=\left\{\boldsymbol{x}(\varphi, \theta, r): r=\sum_{l=0}^{N_{A}} \sum_{m=-l}^{l} a_{l m}^{j} Y_{l m}(\varphi, \theta)\right\}
$$

where $(\varphi, \theta, r)$ are the azimuth, elevation, and distance to the origin. The functions $Y_{l m}$ are the spherical harmonics [14]. $N_{A}$ is the order of the decomposition and determines the number of terms that equal $\left(N_{A}+1\right)^{2}$. The coefficients $a_{l m}^{j}$ are chosen to minimize the functional

$$
J\left(a_{l m}^{j}\right)=\sum_{n=1}^{N_{1 j}}\left(r_{n}^{j}-\sum_{l=0}^{N_{A}} \sum_{m=-l}^{l} a_{l m}^{j} Y_{l m}\left(\varphi_{n}^{j}, \theta_{n}^{j}\right)\right)^{2}
$$

where the points $\boldsymbol{x}_{n}^{j}=\left(\varphi_{n}^{j}, \theta_{n}^{j}, r_{n}^{j}\right), n=1, \ldots, N_{1 j}$, are the sets of known points of the surfaces $R_{j}^{1}, j=1, \ldots, N_{I}$.

Repeating this procedure, we find the coefficients $b_{l m}^{j}$ and $c_{l m}^{j}$ of the spherical harmonics decomposition of the skull-scalp $R_{j}^{2}$ and scalp-air $R_{j}^{3}$ interfaces. Then, for each observed model, we form a vector with the coefficients of the different interfaces, $d^{j}=\left[a_{00}^{j}, \ldots, a_{N_{A} N_{A}}^{j}, b_{00}^{j}, \ldots\right.$, $\left.b_{N_{A} N_{A}}^{j}, c_{00}^{j}, \ldots, c_{N_{A} N_{A}}^{j}\right]^{T}$. Next, we compute the mean value $\bar{d}$ of the coefficients among the different individuals to obtain the standard surfaces $S_{0}^{q}, q=1,2,3$. We arrange the variations of the coefficients $d^{j}-\bar{d}$ in a matrix $A$ of size $3\left(N_{A}+1\right)^{2} \times N_{I}$. Each column $j$ of the matrix $A$ contains the coefficients of the spherical harmonics decomposition of the surfaces $R_{j}^{q}$. A singular value decomposition of matrix $A$ yields

$$
A=U D V^{T}
$$

where $D$ is a diagonal matrix formed by the singular values of matrix $A$. Note that there are $N_{I}-1$ nonzero singular values since the rows of $A$ have zero mean value. We then define the matrices $B=U D^{-} / \sqrt{N_{I}}, W=\sqrt{N_{I}} V^{-T}$ so that $A=B W$, where $D^{-}$and $V^{-}$are formed by the $N_{I}-1$ columns of $D$ and $V$ associated with nonzero singular values. The size of matrix $B$ is then $3\left(N_{A}+1\right)^{2} \times\left(N_{I}-1\right)$, and the size of matrix $W$ is $\left(N_{I}-1\right) \times N_{I}$. The $j$ th column of $B$ can be interpreted as the spherical harmonics decomposition coefficients of surfaces $S_{i}^{q}, q=1,2,3$, that can be combined to represent the original surfaces. The surfaces $S_{i}^{q}$ are then obtained by replacing the $a_{l m}^{j}$ coefficients in (2) by the elements in the corresponding column of $B$. Matrix $W$ is a weight matrix that indicates how to combine the surfaces $S_{i}^{q}$ to get the original surfaces $R_{j}^{q}$. The columns of $B$ corresponding to the largest singular values have more importance in the description of the surfaces, and it is possible to obtain good reconstructions of the surfaces $R_{j}^{q}$ with a reduced number $N_{c}$ of surfaces components $S_{i}^{q}$, as will be seen later. We have then

$$
R_{j}^{q} \approx S_{0}^{q}+\sum_{i=1}^{N_{c}} S_{i}^{q} w_{i j}, \quad q=1,2,3
$$

where $w_{i j}$ are the elements of $W$. By assuming that these coefficients are samples of uncorrelated random variables $\chi_{i}$, we get the proposed expansion (1) of the the random surfaces $\mathcal{S}^{q}$. This algorithm produces coefficients $w_{i j}$ that could come from uncorrelated random variables $\chi_{i}$ with zero mean and unit variance, which we will group in a random vector $\chi$. Assuming that the samples also come from a normal distribution $\chi \sim \mathcal{N}(0, I)$, the procedure yields a decomposition of the random surface $\mathcal{S}^{q}$ as a combination of deterministic surfaces $S_{i}^{q}$ and independent 
random coefficients. The choice of a normal distribution (or any other distribution with probability density function with infinite support) is questionable because it will lead to a nonzero possibility of models in which the interfaces intersect each other, which is physically impossible. We expect that this probability will be small since the surface components are derived from real head models. If an intersection between surfaces is found, an ad hoc local modification of the surfaces would be applied to avoid it.

The surfaces $S_{0}^{q}, q=1,2,3$, represent the average of all the subjects under consideration. These surfaces define what we will refer to as a standard head model, and we will use it later for solving the inverse problem when the true shape of the head is unknown. The electrode positions in this average model are obtained by projecting the average position of the electrodes on the outer surface.

We also built the random head model using local expansions for defining the surface components, i.e., with spline interpolation, e.g., [15], instead of spherical harmonics. The resulting shape of the surface components was essentially the same, especially for the first and most important surface components.

\section{A. Example of a Random Head Model}

With the described procedure, we obtained a random model for the shape of the head based on $N_{I}=30$ different adult individuals. The Curry software (Compumedics Neuroscan, Charlotte, NC) was used to load each individual's 3-D T1-weighted structural MRI scan and automatically segment the shape of the outer skin, outer skull, and inner skull surfaces [16]. Each surface was then represented by $N_{p} \approx 2000$ equally distributed points [17]. The spherical harmonics expansion was of order $N_{A}=19$, giving a total of 400 coefficients for each surface. Extreme care should be taken while working with high-order spherical harmonics due to numerical instabilities [18]. If the order of the expansion is too high, overfitting to the points $\boldsymbol{x}_{n}^{j}$ could occur, increasing the overall error of the approximation. With our choice of $N_{A}=19$, the difference between the true surfaces and their expansions has an rms value under half a millimeter, with

$$
\mathrm{RMS}=\sqrt{\frac{1}{N_{p}} \sum_{n=1}^{N_{p}}\left(r_{n}-\hat{r}_{n}\right)^{2}}
$$

where $r_{n}$ is the distance to the origin of the points $\boldsymbol{x}_{n}$ and $\hat{r}_{n}$ the distance to the origin of the point in the approximated surfaces with the same azimuth and elevation angles as $\boldsymbol{x}_{n}$.

An average surface and $N_{I}-1=29$ surface components were obtained for each head model surface. To test the normal distribution of the random coefficients $\chi_{i}$, we performed a Kolmogorov-Smirnov test on the coefficients $w_{i j}$ in (5). We performed the test for each of the 29 coefficients $(i=1, \ldots, 29)$ with sample size $30(j=1, \ldots 30)$ at $5 \%$ significance level, and found no evidence $(p>0.35)$ for rejecting a normal distribution, except for the smallest surface component $\chi_{29}(p=0.005)$. Since this last coefficient is associated to a surface component

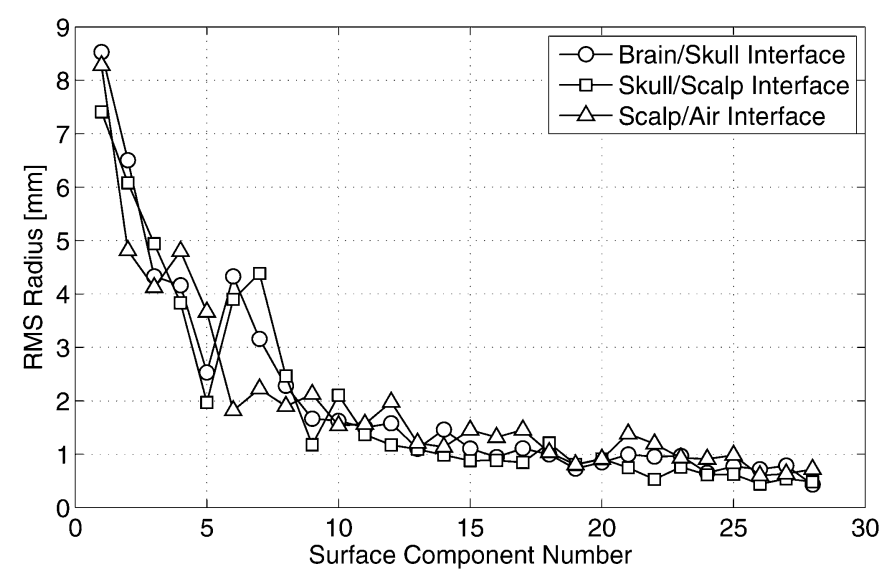

Fig. 1. rms contribution of the surface components of the brain-skull, skullscalp, and scalp-air interfaces.

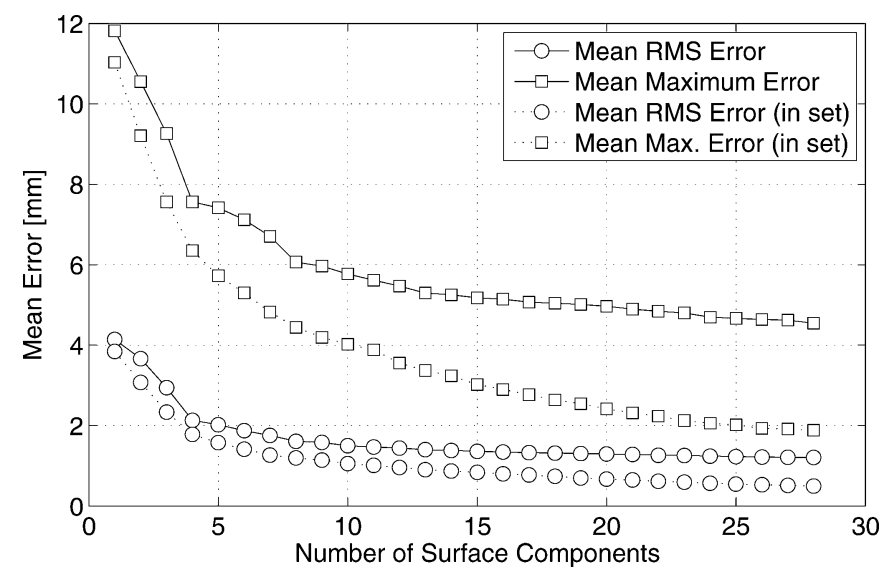

Fig. 2. rms error and maximum error in the representation of the head surfaces as a function of the number of surface components. The error was computed on the upper hemisphere of each of the 30 head models when represented by the surface components derived from the remaining 29. The mean error of the 30 cases is shown for the brain-skull interface. The dotted lines show the mean rms and maximum errors in the representation of head models used in the construction of the surface components.

with a maximum radius smaller than $1 \mathrm{~mm}$, we regard this nonGaussian behavior as negligible.

In Fig. 1, we show the rms value of the radius of the different surface components. The figure shows that only a few components have an important contribution to the overall shape, supporting the idea that a reasonable approximation is possible with a limited number of surface components.

Next, we want to determine the extent to which our random model can represent the shape of heads not used in its construction and the number of surface components needed to attain a good approximation. We performed a leave-one-out crossvalidation analysis [19], determining the difference between the actual head model of each of the 30 individuals and the best approximation to these models built based on the principal surface components, constructed by the remaining 29 head models. The error measure is the rms error (6) between the surfaces and their approximations at points $\boldsymbol{x}_{n}^{j}$.

In Fig. 2, we show the rms and maximum error between the true brain-skull interface and its reconstructions; for the 
skull-scalp and scalp-air interfaces, the errors are slightly smaller. The figure also shows in dotted lines the average of the error in the representation of the head models that were used to build the random model. The figure shows the results as a function of the number $N_{c}$ of surface components included in the random head model; this number may vary between $N_{c}=1$ (average model and the first surface component) and $N_{c}=N_{I}-1=29$. We see in the figure that beyond a certain number of surface components, the effect of increasing $N_{c}$ is not very significant. Note that although the proposed procedure minimizes the rms error, the maximum error between each surface and its representation with surface components also decreases when more surface components are used in the model. The maximum error has values between 4 and $5 \mathrm{~mm}$ for a large number $\left(N_{c}>16\right)$ of surface components, as seen in the figure.

The figure also shows that for a number of surface components approaching $N_{I}-1$, the rms dotted line tends to a value under $0.5 \mathrm{~mm}$, which is the error of the expansion in spherical harmonics used to describe the observed models. Since the order of this expansion is finite, details of high spatial frequency are lost in the representation. It is important to note that if the head model differs from the real shape of the order of $1 \mathrm{~mm}$, the effect on the inverse problem solution is not significant [9].

The resulting random head model has a mean value of the outer surface radius of $85 \mathrm{~mm}$ and variations among individuals of $4 \mathrm{~mm}$ standard deviation. The mean scalp thickness is $8.6 \mathrm{~mm}$ with $1.8 \mathrm{~mm}$ standard deviation among individuals, and the mean skull thickness of $6.2 \mathrm{~mm}$ with standard deviation among individuals below $0.9 \mathrm{~mm}$.

\section{B. Source Model}

In this paper, we adopt a dipolar source model. Such a model represents some sources of interest [20] or may be used to obtain results for distributed sources by convolution.

The coordinates of the source position are given by its azimuth and elevation in the coordinate system defined at the beginning of this section for the alignment of the head models. The third coordinate is the depth of the source, measured as the distance between the source and the surface of the brain at the same azimuth and elevation. This choice of coordinates keeps the source depth constant when the shape of the head varies. In this way, we are certain that the source will always be inside the brain, which would not be the case if the third coordinate was, e.g., the distance from the source to the origin of the coordinate system. Another possibility for establishing a correspondence among the source positions in the individual models would be to use anatomically equivalent sources, instead of geometrically equivalent ones. However, establishing anatomical equivalences among individuals is not a trivial task, and it is beyond the scope of this paper.

Note that this specification of the source position relative to the surface of the brain seems favorable for EEG, since the electrode positions also vary with the shape of the head. For the MEG helmet, on the other hand, the position of the coils is fixed in the absolute coordinate system defined by the fiducial points, and variations of the shape of the head would change the relative position between the magnetometers and the source.

\section{FORWARD PROBLEM}

In this section, we show how to solve the EEG and MEG forward problems when the head model has random geometry. The forward problem solutions will also be random since they depend on the geometry of the head. We characterize the forward problem solution, validate the proposed method, and present some results for the random head model of Section II-A.

\section{A. Deterministic Solution}

For solving the deterministic forward problem, we adopt the boundary elements method (BEM), with triangular elements and linear variations over the elements [21]. The use of BEM for solving the EEG/MEG forward problem is widespread [22], [23]; the surfaces are tesselated in small triangular elements and a linear variation of the electric potential is assumed over each element. The integral formulation of the forward problem is then reduced to a linear system that must be solved to obtain the electric potential at the nodes, i.e., at the $N_{N}$ vertices of the elements, and magnetic field at the sensor positions. To avoid numerical problems due to the low electric conductivity of the skull, we use the isolated problem approach (IPA) [24] that solves the EEG forward problem in two steps: first considering a null conductivity for the skull and then modifying the result according to the true value of the skull conductivity.

We restrict our analysis to one-time snapshot; hence, the solution of the EEG and MEG forward problems is given by the vectors $\phi$ and $v$ formed by the electric potential and magnetic field components in the respective sensors.

Under these conditions, the EEG and MEG forward problem solutions are given by

$$
\begin{aligned}
& \boldsymbol{\phi}=E \boldsymbol{\phi}_{S} \\
& \boldsymbol{v}=M \boldsymbol{\phi}_{S}+\boldsymbol{v}_{S}
\end{aligned}
$$

where the linear operators $E$ and $M$ are related to the geometry and electric conductivity of the head model, the vector $\phi_{S}$ is the electric potential at the nodes of the innermost surface that would be generated by the source if the media were homogeneous and infinite, and the vector $\boldsymbol{v}_{S}$ is the measured component of the magnetic field generated by the primary current density. Note that even though the number of rows of matrices $E$ and $M$ is equal to the number of electric potential and magnetic field sensors, the number of operations needed for computing these matrices is proportional to $N_{N}^{2}$. For a detailed surface tessellation with several thousands nodes per surface, this represents an important computational load.

\section{B. Random Head Model Solution}

To analyze the effect of the head shape variability on the forward problem solution, we must solve the problem with the random head model presented previously. Since the forward problem solutions depend on the geometry of the head model, they will also be random. We will denote these random forward problem solutions as $\phi(\chi)$ and $\boldsymbol{v}(\chi)$, where $\chi$ is the normalized Gaussian random vector used in the random head model. 
One possibility would be to use a "brute force" approach, running MCSs to obtain statistics of the forward problem solution. The drawback of this approach is the high computational load it requires; a large number of samples of the random head model is necessary to obtain reliable statistics, and each sample involves the assembling of the matrices associated to the forward problem solution. Instead, we perform a PCE [12], [13] of the random vectors $\phi(\chi)$ and $v(\chi)$. This enables us to obtain a good approximation of the covariance matrices $\operatorname{Cov}\{\phi(\chi)\}$ and $\operatorname{Cov}\{\boldsymbol{v}(\boldsymbol{\chi})\}$, which depend on the source parameters and are of uttermost importance in determining the effect of the random geometry on the inverse problem solutions.

A PCE of any finite-variance random variable $\alpha$ is a series expansion

$$
\alpha=\sum_{k=0}^{\infty} \alpha_{k} g_{k}(\chi)
$$

where $\alpha_{k}$ are deterministic coefficients, $g_{k}(\cdot)$ are multidimensional Hermite polynomials, and $\chi \sim \mathcal{N}(0, I)$ is a normal random vector. The multidimensional polynomials $g_{k}(\cdot)$ are obtained as product of 1-D polynomials $h_{k}(\cdot)$. The first 1-D Hermite polynomials are given by $h_{0}(x)=1, h_{1}(x)=x$, $h_{2}(x)=x^{2}-1$. See, e.g., [25] for information on how to obtain higher degree Hermite polynomials. The degree of the multidimensional polynomial is the sum of the degree in each dimension; thus, if $\chi$ in (9) has $N$ elements, there will be $N$ first-degree Hermite polynomials, $N(N+1) / 2$ second-degree Hermite polynomials, etc. We assume that the index $k$ grows with the degree of the polynomials; the ordering among the polynomials of the same degree plays no role, and thus, is arbitrary. The Hermite polynomials are orthogonal with respect to the Gaussian measure, and form a basis of the Hilbert space of finite-variance random variables [13], [26], with inner product given by the expectation $\mathbb{E}_{\chi}\{\cdot\}$. Thus, the coefficients of the PCE are computed as $\alpha_{k}=\mathbb{E}\left\{\alpha g_{k}(\chi)\right\}$.

The PCE is directly extended to a random vector, and thus, it can be applied to the EEG forward problem solution. Then, an approximation to the EEG forward problem solution is given by the first terms of the PCE

$$
\phi(\chi) \approx \sum_{k=0}^{K} \phi_{k} g_{k}(\chi)
$$

where $\phi_{k}$ are the deterministic vector coefficients that must be found. Once they are known, it is possible to obtain the first- and second-order moments of the solution vector; note that since the random variables $g_{k}(\chi)$ have zero mean (except $g_{0}(\chi)=1$ ), the first moments of the solution are

$$
\begin{aligned}
\mathbb{E}\{\boldsymbol{\phi}(\boldsymbol{\chi})\} & =\boldsymbol{\phi}_{0} \\
\mathbb{E}\left\{\boldsymbol{\phi}(\boldsymbol{\chi}) \boldsymbol{\phi}^{T}(\boldsymbol{\chi})\right\} & =\sum_{k=0}^{\infty}\left\|g_{k}\right\|^{2} \boldsymbol{\phi}_{k} \boldsymbol{\phi}_{k}^{T} \\
& \approx \sum_{k=0}^{K}\left\|g_{k}\right\|^{2} \boldsymbol{\phi}_{k} \boldsymbol{\phi}_{k}^{T}
\end{aligned}
$$

and since we use normalized Hermite polynomials $\left\|g_{k}\right\|=1$.
The deterministic coefficients of the PCE are given by $\phi_{k}=\mathbb{E}\left\{g_{k}(\boldsymbol{\chi}) \boldsymbol{\phi}(\boldsymbol{\chi})\right\}, k=0, \ldots, K$. These expectations are multidimensional integrals that we evaluate numerically. Then

$$
\boldsymbol{\phi}_{k} \approx \sum_{n=1}^{N_{\mathrm{ev}}} h_{n} g_{k}\left(\boldsymbol{\chi}_{n}\right) \boldsymbol{\phi}\left(\boldsymbol{\chi}_{n}\right)
$$

where the proper values for the weights $h_{n}$ and the evaluation points $\chi_{n}$ are selected according to the integration algorithm. The domain of integration is infinite; hence, the adequate quadrature algorithm is the Gauss-Hermite quadrature [25]. But a direct extension of a 1-D quadrature algorithm to a higher dimensional space is impractical because the number of evaluation points $N_{\text {ev }}$ grows exponentially with the number of dimensions. We instead use a Smolyak or sparse grid algorithm [27]-[29]. A Smolyak quadrature of order $o$ is a combination of lowdimensional quadrature algorithms yielding exact integration for multidimensional polynomials of total degree up to $2 o-1$, and for which the number of evaluation points grows polynomically with the number of dimensions.

The use of 13 to obtain the PCE coefficients $\phi_{k}$ requires the computation of $N_{\mathrm{ev}}$ forward problem solutions $\phi\left(\chi_{n}\right)$, each one being a solution of the deterministic forward problem III-A. A PCE of the MEG forward problem solution can be obtained in the same way.

\section{Validation}

Next, we validate the proposed methods for solving the forward problem when the head model is random. First, we find the right values for parameters involved in the forward problem solution, such as the number of surface components and the numerical integration order needed to obtain accurate results. Then, we compare the forward problem solution with an MCS for a small-size problem.

We solved the EEG and MEG forward problems for the random head model of the previous section. Each surface was tesselated in 1280 triangular elements, resulting in a total number of nodes $N_{N}=1926$. The assumed electric conductivity for the brain and scalp was $0.33 \mathrm{Sm}^{-1}$, and for the skull $0.022 \mathrm{Sm}^{-1}$. This conductivity ratio of $1 / 15$ is supported by recent studies [30]-[32]. The forward problem was solved for 69 electrodes and 160 radial magnetometers.

The dipolar sources we adopt in this paper are all of $20 \mathrm{nAm}$ intensity, with the dipole moment tangential to the surface of a sphere centered at the origin, and in a plane containing the $z$-axis. In this section, we analyze the results for 106 dipolar sources regularly distributed on the upper hemisphere of the brain, at $10 \mathrm{~mm}$ depth under the brain-skull interface.

First, we tested the numerical integration scheme. For this test, the number of principal surface components $N_{c}$ does not play an important role; so we adopt $N_{c}=4$ to speed up the computations, leading to a number of integration points of $N_{\mathrm{ev}}=1,9,45,165,494$, and 1278 for integration order $o=1$ to 6 . The results obtained for $o=6$ were used as the reference. We quantify the error using the relative difference 


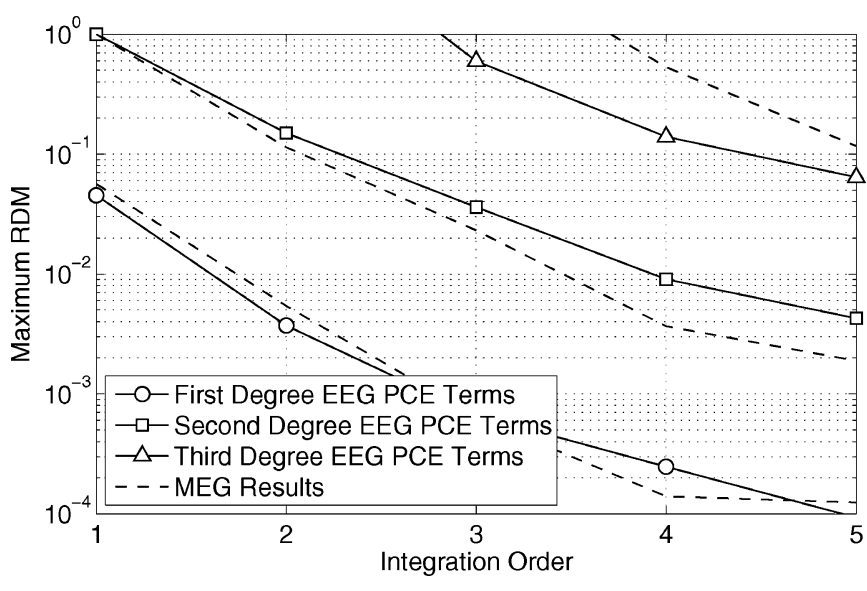

Fig. 3. RDM as a function of the numerical integration order. The reference is the result for order 6. RDM of the coefficients of the PCE of the EEG and MEG forward problem solution. Maximum RDM among all the PCE terms of the same order and among 106 different sources in the upper hemisphere of the head at $10 \mathrm{~mm}$ depth.

TABLE I

MaXimum RMS VAlue of THE COEFFICIENTS OF DiFFERENT DEGREeS IN THE EEG AND MEG ForWARD PROBLEM SOLUTION PCE

\begin{tabular}{|c|c|c|c|c|}
\hline \multirow{2}{*}{ Degree } & \multicolumn{2}{|c|}{ EEG $[\mu V$ RMS $]$} & \multicolumn{2}{c|}{ MEG $[f T$ RMS $]$} \\
\cline { 2 - 5 } & Radial & Tang. & Radial & Tang. \\
\hline 0 & 1.672 & 1.379 & 11.5 & 75.3 \\
1 & 0.166 & 0.138 & 6.1 & 9.9 \\
2 & 0.027 & 0.023 & 0.8 & 1.8 \\
3 & 0.005 & 0.005 & 0.2 & 0.5 \\
\hline
\end{tabular}

measure (RDM) [23] given by

$$
\mathrm{RDM}=\frac{\left\|\phi_{k}^{(0)}-\phi_{k}^{(6)}\right\|_{2}}{\left\|\phi_{k}^{(6)}\right\|_{2}}
$$

where $\phi_{k}^{(o)}$ denotes the $k$ th coefficient of the PCE, computed with (13) using a numerical integration order $o$.

In Fig. 3, we show the error as a function of the integration order $o$. We plot the maximum RDM among all the sources and all the terms of the same degree in the PCE. Full lines are used for the EEG and broken lines for the MEG forward problems. As expected, the error decreases with the integration order. The error is higher for terms related to higher degree Hermite polynomials in the PCE (9), because these polynomials appear in the integrand, and the numerical integration algorithm is exact only for polynomials of total degree up to $2 o-1$.

We also computed the contribution of the terms of different degree in the PCE. Table I shows the rms value of the forward problem solution; the value corresponds to the mean value among the 106 sources mentioned earlier and the maximum among the terms of the same degree in the PCE. The EEG results are measured in microvolts and the MEG results in femto Tesla (fT). It is clear that the contribution of higher degree terms of the PCE is small compared to the expected value (degree 0) and first degree terms, and could be neglected.

Next, we study the effect of increasing the number of surface components $N_{c}$ for the random head model. To quantify the effect of the random head model on the forward problem solution, we use the signal to variations ratio (SVR), defined as the ratio

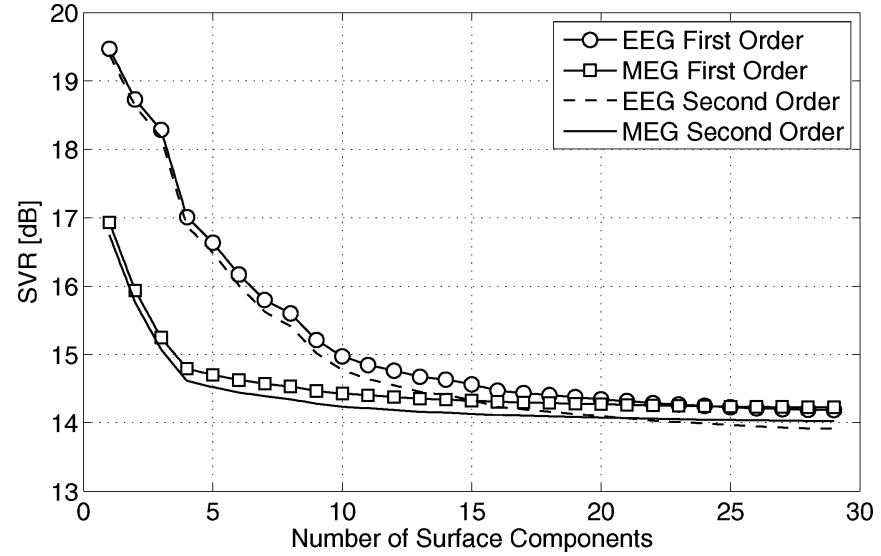

Fig. 4. SVR of the EEG and MEG forward problem solutions as a function of the number of surface components included in the random head model. Results for first- and second-order PCEs are shown.

between the norm of the expected value of the forward problem solution and the energy of the deviations with respect to this value. For a random vector expressed by its PCE (9), the SVR is in logarithmic scale

$$
\mathrm{SVR}=10 \log _{10} \frac{\|\mathbb{E}\{\boldsymbol{\phi}(\boldsymbol{\chi})\}\|_{2}^{2}}{\| \operatorname{Cov}\left\{(\boldsymbol{\phi}(\boldsymbol{\chi})\} \|_{2}\right.}=10 \log _{10} \frac{\left\|\phi_{0}\right\|_{2}^{2}}{\sum_{k=1}^{K}\left\|\phi_{k}\right\|_{2}^{2}} .
$$

Note that while the RDM is used to compare two deterministic vectors, the SVR quantifies the variations of a random vector and is similar to a signal to noise measure, where the "noise" is due to the variations of the head shape.

In Fig. 4, we show the mean value of the SVR among the different sources for first- and second-degree PCE. Taking into account the second-degree terms of the PCE decreases the SVR in less than $0.3 \mathrm{~dB}$, so it is possible to obtain a reasonable approximation to the forward problem solution with a firstdegree PCE. It is also possible to note in the figure that the most important contribution comes from the first surface components, as expected, since they correspond to larger variations of the head shape. The figure also shows that even though the EEG and MEG mean SVR are almost equal for these sources, the MEG results depend only on a few surface components (around 5), while the EEG result has significant contributions from more surface components (around 12). This suggests that the EEG forward problem requires a more detailed description of the surfaces.

To validate the forward problem solution, we performed a traditional MCS, solving the forward problem for samples of the random head model, with five runs of 500 samples, for 106 tangentially oriented dipolar sources at $10 \mathrm{~mm}$ depth. We compared the MCS results with the results obtained by the proposed methodology. We chose $N_{c}=15$ surface components to build the random head model, a first-order PCE, and integration order $o=3$, resulting in $N_{\mathrm{ev}}=496$ evaluation points for computing the coefficients (13) of the PCE. These parameter values were chosen because, as seen in Table I and Fig. 4, such a model incorporates almost all the randomness observed in the different individuals, and as shown in Fig. 3, the error of the 
numerical integration is negligible in this case (first-order PCE and integration order $o=3$ ).

The computational burden of the PCE approach is exactly equivalent to the solution of $N_{\mathrm{ev}}$ deterministic forward problems, as discussed at the end of Section III-B. Thus, it is the same as an MC run of length $N_{\mathrm{ev}}$. We performed an MCS with runs of length 500, and compared the results with the PCE solution of the random head model adopted in the previous paragraph $\left(N_{\mathrm{ev}}=496\right)$. The first-order PCE leads to a forward problem solution with normal distribution, completely characterized by its expected value and covariance matrix. Hence, we will compare only the first- and second-order moments of the PCE and MCS solutions. The mean value of the forward problem solution of both approaches have an RDM value lower than 0.005 , which is negligible from any practical point of view. To compare the covariance matrices of the forward problem solutions, which describe the variations due to the random head model, we compute the relative error of the Frobenius norm of the difference between pairs of covariance matrices. The PCE covariance matrix is computed with (12), and an approximate value of its error is given by the difference between the firstorder PCE results and the second-order PCE results, which is of 5\%. The MCS produces different estimates of the covariance matrix for each run, and the difference between them is around $18 \%$, between three and four times larger than the PCE error. The expected value of the relative difference of the Frobenius norm between MCS runs is inversely proportional to the square root of the number of trials; hence, to achieve the same error level as PCE, the number of MCS trials and its computational burden, is between 10 and 15 times larger.

\section{Results}

For the random head model validated in the previous section $\left(N_{c}=15\right.$, first-order PCE, integration order $\left.o=3\right)$, we computed the SVR for tangentially oriented dipolar sources at $10 \mathrm{~mm}$ depth. In Fig. 5, we show the SVR of the EEG and MEG forward problem solution as a function of the dipole position. The figures also show the tesselation of the standard shape of the brain-scalp interface. We observe that the variations of the SVR with the source position are in the range of $3 \mathrm{~dB}$, for both the EEG and MEG forward problems.

Comparing the EEG and MEG results, we see that the effect of the random geometry on the forward problem is slightly larger for MEG than for EEG, even though for the MEG forward problem this effect is produced by a lower number of surface components, as seen in Fig. 4. The MEG forward problem solution (8) is formed by two terms: one related to the volumetric currents and the other due to the primary current density. We found that the contribution of the second term to the variance of the results is more than four times larger than the contributions due to the volumetric currents, at least for the tangentially oriented sources under analysis. This indicates that the main source of variations in the MEG forward problem result is not due to the variations in the shape of the volume conductor but due to the variations of the relative position between the source and the magnetometers.

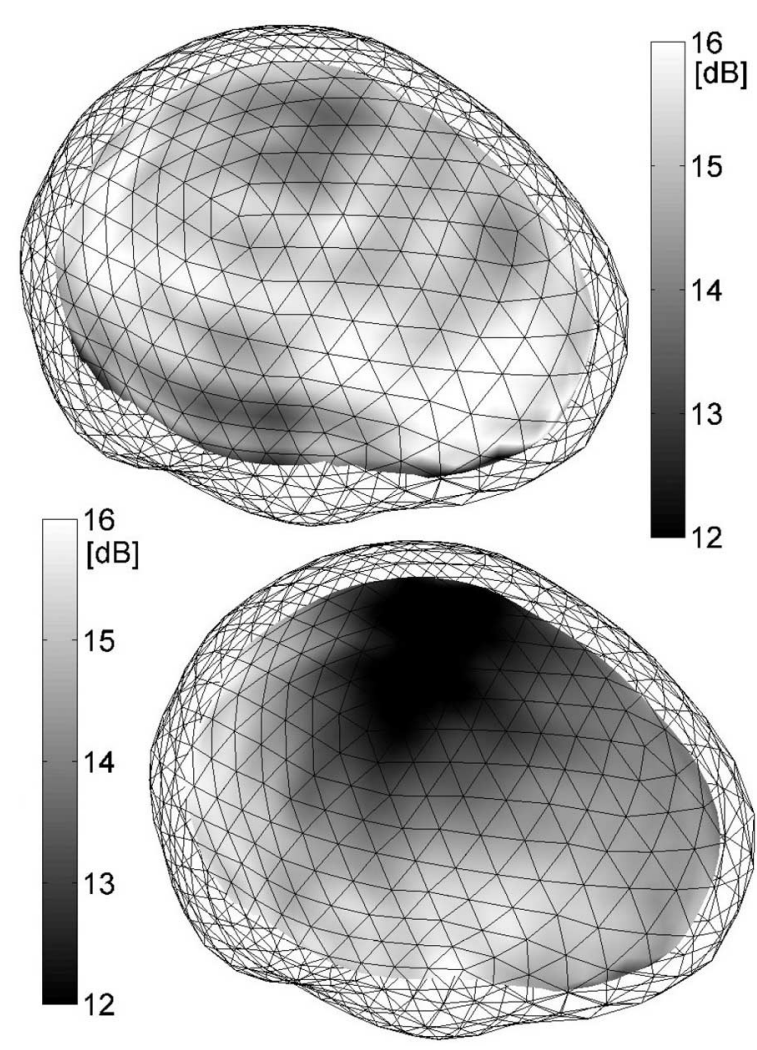

Fig. 5. SVR of the forward problem solution due to the random geometry of the head model. The random model of the head includes 15 surface components. The results correspond to a tangentially oriented dipolar source at a depth of $10 \mathrm{~mm}$ under the surface of the brain. The mesh corresponding to the average brain/skull interface is also shown. The gray level indicates the SVR for a source located at each point. (Top) EEG results. (Bottom) MEG results.

TABLE II

EEG AND MEG ForWARd PRoblem Solution Statistics For RAdial $\left(D_{r}\right)$ AND TANGENTIAL $\left(D_{t}\right)$ DipOLES

\begin{tabular}{|l|c|c|c|c|c|c|c|}
\multicolumn{2}{c}{} & \multicolumn{3}{c}{ SVR $[\mathrm{dB}]$} & \multicolumn{2}{c}{$\overline{\mathrm{RDM}}$} & \multicolumn{2}{c|}{$95 \%$ RDM } \\
\cline { 3 - 9 } \multicolumn{2}{c|}{} & $D_{r}$ & $D_{t}$ & $D_{r}$ & $D_{t}$ & $D_{r}$ & $D_{t}$ \\
\hline \multirow{2}{*}{$\begin{array}{l}\text { Random head } \\
\text { shape }\end{array}$} & EEG & 13.8 & 15.0 & 0.18 & 0.16 & 0.39 & 0.31 \\
\cline { 2 - 9 } & MEG & 3.0 & 13.0 & 0.62 & 0.18 & 1.24 & 0.41 \\
\hline \hline \multirow{2}{*}{$\begin{array}{l}\text { Electronic } \\
\text { Noise }\end{array}$} & EEG & 12.9 & 11.6 & 0.22 & 0.26 & 0.26 & 0.30 \\
\cline { 2 - 9 } & MEG & -9.4 & 8.2 & 2.93 & 0.39 & 3.22 & 0.42 \\
\hline
\end{tabular}

In Table II, we show more results for dipolar sources that we will denote by $D_{r}$ and $D_{t}$, located on the left frontal lobe, with azimuth $-\pi / 5 \mathrm{rad}$ and elevation $\pi / 4 \mathrm{rad}$. The source $D_{r}$ has a radial orientation and $20 \mathrm{nAm}$ intensity, and $D_{t}$ has a tangential orientation, with dipolar moment $(11.4,-8.3,-14.1) \mathrm{nAm}$, i.e., also $20 \mathrm{nAm}$ intensity. Later on, we will use these sources in other examples. The table shows the SVR, the mean value of the RDM among different samples of the random forward problem, and the $95 \%$ quantile of the RDM among samples for dipoles $D_{r}$ and $D_{t}$ at $10 \mathrm{~mm}$ depth. We also include typical results for additive electronic noise, assuming the standard deterministic head model and a Gaussian distribution of the electronic noise, independent between sensors, with a typical standard deviation of $0.4 \mu \mathrm{V}$ for EEG sensors and $32 \mathrm{fT}$ for MEG [33]-[35]. Observe that the noise term does not include background brain activity. It is also important to note that the results for the electronic noise 
depend on the source intensity, while the relative effect of the random head model is independent of the source intensity. This is so because all the terms in the PCE are proportional to the source intensity, and as a result, the variation of the intensity cancels out in the computation of the SVR or any other relative error measure.

We see that the effect of the random geometry of the head model in the forward problem solution is smaller than the effect of the electronic noise, but the former is spatially correlated. Hence, we can draw no conclusions yet regarding the effect of the inverse problem. A deeper analysis is necessary, and we perform it in the next section.

\section{INVERSE PROBLEM}

In this section, we study the effect of the random geometry of the head model in the inverse problem. We want to determine the error in the estimation of the source position with the standard head model, when the true geometry is a sample of the random head model. We study the localization error due only to the random geometry, i.e., when there are no noise sources present.

\section{A. Method}

For any dipolar source in the brain, we want to obtain a statistical description of the localization error when the inverse problem is solved with the standard head shape. This statistical description consists of the first- and second-order moments, or an approximation to the distribution. An attempt to express the localization error in any spatial direction with a PCE and apply the same idea as for solving the forward problem showed that a high-order PCE is needed. This leads to a large number of evaluation points for computing the expectations numerically, yielding the procedure impractical.

We then resort to MCSs, generating the samples of the forward problem solution from (10). The algorithm is the following: for any given dipolar source compute a sample of the forward problem solution $\phi_{m}$, which we will call the measurement. Then, minimize the difference $\left(L_{2}\right.$ norm) between the measurements and the forward problem solution of a dipolar source with the deterministic standard head model. The parameters of the dipole that achieves this minimization are the solution of the inverse problem. The minimization algorithm we use is a simple steepest descent method, where the best-fitting dipole moment is computed at every test location. This algorithm performs adequately in the mentioned conditions, i.e., one dipolar source and no additive noise present.

\section{B. Validation}

To validate the proposed methodology for characterizing the inverse problem solution, we solve the inverse problem for the dipolar sources $D_{r}$ and $D_{t}$ defined previously, at a depth of $10 \mathrm{~mm}$.

First, we perform an MCS; we generate a sample of an independent normal random vector $\chi$, and build one sample of the random head model with (1). We solve the forward problem for that head model, obtaining the vector of electric potential or magnetic field that would be present at the sensor locations. With these data, we solve the inverse problem assuming the standard head model. Repeating this 1000 times, we form a vector $d_{\mathrm{MC}}$ with the distance between the true source position and the estimated one in each case.

Then, we form a second vector $d_{\mathrm{PC}}$ with the distance between true and estimated source positions when the forward problem solution is given by the PCE. In this case, we also start from an independent normal random vector $\chi$ and use (10) to obtain the forward problem solution for the corresponding head shape, without actually computing the surfaces of the model. Again, we solve the inverse problem assuming the standard head model and repeat the procedure 1000 times.

We performed a Kolmogorov-Smirnov test to determine if the samples of $d_{\mathrm{MC}}$ and $d_{\mathrm{PC}}$ could be samples from the same random variable. The test at $1 \%$ significance level showed no evidence for rejecting the null hypothesis that the samples came from the same variable, both for the EEG $(p=0.012)$ and MEG $(p=0.105)$ inverse problems.

The results of the test show that a very good approximation of the inverse problem solution is obtained when solving the forward problem with the proposed procedure. The approximation is good in the sense that it produces inverse problem solutions based on the standard head model that are similar to the results of a MCS.

\section{Results}

For the random head forward problem solution obtained in the previous section, we computed the geometric distance between the true dipole positions and the estimated positions when solving the inverse problem with the standard head model, for tangentially oriented dipolar sources at $10 \mathrm{~mm}$ depth. We repeated this for 1000 forward problem samples and obtained an error measure for the inverse problem as the $95 \%$ quantile of the distance between the real and estimated source positions. Fig. 6 shows the results for the EEG and MEG inverse problem solution as a function of the dipole position. We see in the figure that the variations of the localization error for different positions of the source are around a factor 2 for the EEG and MEG results, as was the case for the forward problem results. We also observe that the effect of the random head geometry is significantly larger on the MEG inverse problem than on the EEG inverse problem.

The localization errors as a function of source depth are shown in Fig. 7. The results correspond to dipole $D_{t}$ at different depths. We observe that error does not vary too much with depth.

The method described in this paper is also useful when there is partial information regarding the shape of the head model. To show an example of this, we built a different random head model for which we assume that the outer surface, or scalpair interface, is known. In this case, the random head model is given by two random fields describing not the absolute shape of scalp and skull but rather the scalp and skull thickness. The head model and forward problem solution are obtained with the methodology described in the previous sections, and the results are shown for dipole $D_{t}$ in Fig. 7. It is possible to see a fourfold 


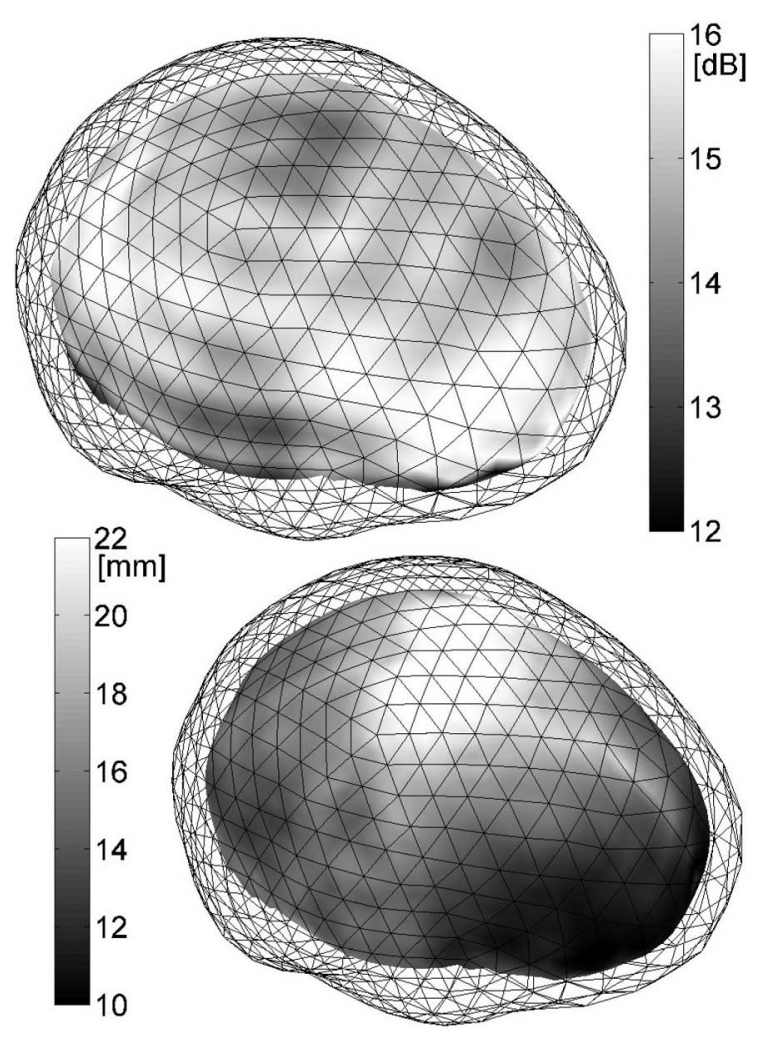

Fig. 6. Localization error of the inverse problem when solved with the average head model, and the true geometry is given by samples of the random head model. The results correspond to a tangentially oriented dipolar source at a depth of $10 \mathrm{~mm}$ under the surface of the brain. The gray level indicates the $95 \%$ quantile of the distance between the true and estimated dipole positions for a source located at each point. (Top) EEG results. (Bottom) MEG results.

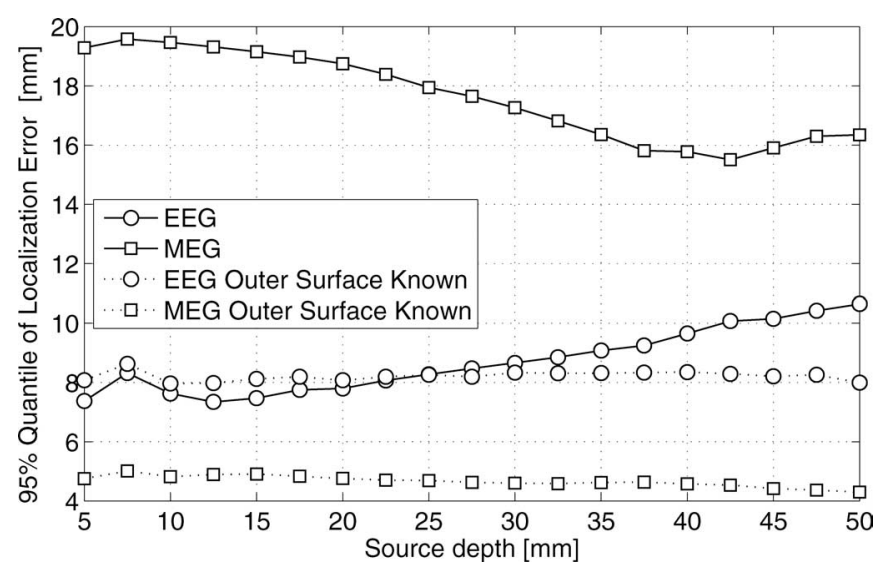

Fig. 7. $95 \%$ quantile of EEG and MEG inverse problem localization errors. The results correspond to a tangentially oriented dipolar source in the left temporal lobe. The results are shown for the random head model with three random surfaces and a random model in which the outer surface is known.

reduction of the error for the MEG results, while the EEG results do not vary significantly.

In Table III, we show a comparison of localization errors obtained for dipoles $D_{r}$ and $D_{t}$ at $10 \mathrm{~mm}$ depth. The table shows the mean value of the localization error, its standard deviation, and the 95\% quantile, computed from an MCS with 1000 runs. We can see that except for the radial dipole MEG result, the
TABLE III

STATISTIC OF THE EEG AND MEG INVERSE PROBLEM LOCALIZATION ERRORS [IN MILLIMETERS]

\begin{tabular}{|c|c|c|c|c|c|c|c|}
\hline & \multicolumn{2}{|c|}{ Mean } & \multicolumn{2}{|c|}{ St.Dev. } & \multicolumn{2}{|c|}{$95 \%$ quantile } \\
\hline & & $D_{r}$ & $D_{t}$ & $D_{r}$ & $D_{t}$ & $D_{r}$ & $D_{t}$ \\
\hline Random head & EEG & 3.8 & 3.5 & 2.6 & 2.1 & 7.8 & 7.2 \\
\hline shape & MEG & 6.8 & 7.1 & 4.3 & 5.3 & 14.5 & 16.6 \\
\hline Outer surface & EEG & 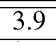 & 3.6 & 2.8 & 2.4 & 8.2 & $\overline{77.6}$ \\
\hline known & $\mathrm{MEG}$ & 2.7 & 2.2 & 1.5 & 1.5 & 5.3 & 4.8 \\
\hline Electronic & EEG & 2.7 & 2.9 & 1.2 & 1.3 & 5.1 & 5.2 \\
\hline Noise & MEG & 27.3 & 2.5 & 18.7 & 1.1 & 62.9 & 4.6 \\
\hline
\end{tabular}

effect of electronic noise in the measurements is smaller than the effect of the random head geometry, even though the effect of the former in the forward problem was lower. This is because the variations due to the electronic noise are not spatially correlated, and hence, easier to differentiate from brain activity. The spatial correlation of the random head shape MEG forward problem results is especially important for the radial dipole; the variations due to the head geometry appear mostly as variations in the amplitude of the magnetic field distribution, while the shape of the distribution is not too distorted. Hence, the localization error is almost equal as for the tangential dipole.

It is important to note that the results shown for the electronic noise are for one-time snapshot, and if a certain dynamics is assumed for the source, the error could be lowered using information from other instants. This is not true for the effect of the random geometry; no error reduction is achieved by combining measurements from different time slots since they all correspond to the same sample of the random head model, i.e., the same individual.

\section{DISCUSSION}

The random head model used in this paper consists of a combination of deterministic surfaces with random coefficients. We built a model based on observed models of different adult individuals and found that a limited number of surface components can represent any head shape with a residual error of a few millimeters. We believe that the use of large databases for the construction of the random model would yield better results, i.e., reduce the residual error by choosing the most representative surface components from a large set of different observed models. With the use of a large database of head models, it would also be possible to build gender- or age-specific random models.

We solved the forward problem with the random head model by finding the first terms of the PCE of the solution. With this expansion, we obtain a reasonable statistical characterization of the effect of the random shape on the forward problem, with a practical amount of computational load. Another option we studied in previous research [36], [37] is to use the stochastic finite-elements method [13] for obtaining the PCE of the forward problem solution, but it involves the solution of a larger linear system, which increases the computational load of the problem.

We showed that a very good approximation of the effect of the random head model on the forward problem solution is given by a Gaussian distribution, which is obtained by the 
first-order terms of the PCE. The deviations from the mean value are spatially correlated and are lower than the electronic noise associated to the sensors.

Regarding the effect of the random geometry on the EEG/MEG inverse problem, we restricted our analysis to the estimation of one dipolar source position by assuming an average head model, and found that the maximum localization error is of the order of $10 \mathrm{~mm}$ for the EEG forward problem and between 15 and $20 \mathrm{~mm}$ for the MEG forward problem. The difference between EEG and MEG results is due to the specification of the source position relative to the brain surface. Nevertheless, the specification of the source position cannot be given in an absolute coordinate system since the source could then lie outside the brain. Also, it is sensible to assume an alignment between the MEG helmet and the head based on the fiducial points (which define the absolute coordinate system). In conclusion, the errors we give for the source position estimation with an average head model are due to the shape variations of the volume conductor for EEG inverse problem, while for the MEG case, the errors are mainly due to the change of the relative position between the source and the MEG helmet. This is supported by the important reduction of the MEG errors when the outer surface of the head is known, as shown in Fig. 7.

With regards to our choice of a head model with three layers of isotropic electric conductivity and a dipolar source model, we believe that it is appropriate for studying the effect of the head shape variations since the results are representative enough. In addition, more exact models, e.g., with anisotropic and inhomogeneous electric conductivity and distributed sources, have too many parameters (conductivity tensor of the tissues, extent, and shape of the sources) that would complicate the interpretation of the effects of the head shape variations.

In this paper, we assumed that only the average head shape is known and used it for solving the inverse problem. An alternative approach may be to consider that the principal surface components are also known. This should lead to a reduction in the errors since it involves some information regarding the structure of the spatial correlation matrix. It is possible to compute the Cramér-Rao bound in this situation, including a term for the bias of the inverse problem solution.

We conclude that the use of an average or standard model for solving the EEG/MEG inverse problems instead of the true head geometry of each individual leads to worst case localization errors under $10 \mathrm{~mm}$ for EEG and $20 \mathrm{~mm}$ for MEG. This value is similar to the results of other studies [10], [11] with deterministic approaches, but in this paper we generalize these results to a large family of head models instead of several cases. Note that this paper does not aim to propose a particular average model, but to quantify the localization errors when such an average model is used. To decide upon the significance of these errors, one should consider other sources of uncertainty in the EEG/MEG inverse problems. The effect of electronic noise of the sensors is well established [35], but there are other important factors that are sometimes overlooked in the literature of inverse problems, notably the background brain activity [38] when working with dipolar source models, and in EEG, the electrode noise related to the electrochemical skin-gel interface, larger than the electronic noise of the sensors [39].

\section{REFERENCES}

[1] J. W. H. Meijs, B. J. ten Voorde, M. J. Peters, C. J. Stock, and F. H. L. da Silva, "On the influence of various head models on EEG's and MEG's," in Functional Brain Imaging, G. Pfurtscheller and F. H. L. da Silva, Eds. Berlin, Germany: Springer-Verlag, 1988.

[2] B. N. Cuffin, "Effects of head shape on EEG's and MEG's," IEEE Trans. Biomed. Eng., vol. 37, no. 1, pp. 44-52, Jan. 1990.

[3] B. Roth, A. Gorbach, and S. Sato, "How well does a three-shell model predict positions of dipoles in a realistically shaped head?," Electroencephalogr. Clin. Neurophysiol., vol. 87, pp. 175-184, 1993.

[4] B. Yvert, O. Bertrand, M. Thévenet, J. F. Echallier, and J. Pernier, “A systematic evaluation of the spherical model accuracy in EEG dipole localization," Electroencephalogr. Clin. Neurophysiol., vol. 102, pp. $452-$ 459, 1997.

[5] S. Baillet, J. J. Rivera, G. Marin, J. F. Mangin, J. Aubert, and L. Garnero, "Evaluation of inverse methods and head models for EEG source localization using a human skull phantom," Phys. Med. Biol., vol. 46, pp. 77-96, 2001.

[6] J. P. Ary, S. A. Klein, and D. H. Fender, "Location of sources of evoked potentials: Corrections for skull and scalp thicknesses," IEEE Trans. Biomed. Eng., vol. BME-28, no. 6, pp. 447-452, Jun. 1981.

[7] B. N. Cuffin, "Effects of local variations in skull and scalp thickness on EEG's and MEG's," IEEE Trans. Biomed. Eng., vol. 40, no. 1, pp. 42-48, Jan. 1993.

[8] G. Huiskamp, M. Vroeijenstijn, R. van Dijk, G. Wieneke, and A. van Huffelen, "The need for correct realistic geometry in the inverse EEG problem," IEEE Trans. Biomed. Eng., vol. 46, no. 11, pp. 1281-1287, Nov. 1999.

[9] N. von Ellenrieder, C. H. Muravchik, and A. Nehorai, "Effects of geometric head model perturbations on the EEG inverse problem," IEEE Trans. Biomed. Eng., vol. 53, no. 2, pp. 249-257, Mar. 2006.

[10] D. van't Ent, J. C. de Munck, and A. L. Kaas, "A fast method to derive realistic BEM models for E/MEG source reconstruction," IEEE Trans. Biomed. Eng., vol. 48, no. 12, pp. 1434-1443, Dec. 2001.

[11] M. Fuchs, J. Kastner, M. Wagner, S. Hawes, and J. S. Ebersole, "A standarized boundary element method volume conductor model," Clin. Neurophysiol., vol. 113, pp. 702-712, 2002.

[12] N. Wiener, "The homogeneous chaos," Amer. J. Math., vol. 60, pp. 897936, 1938.

[13] R. G. Ghanem and P. D. Spanos, Stochastic Finite Elements: A Spectral Approach. New York: Springer-Verlag, 1990.

[14] M. Abramowitz and I. A. Stegun, Handbook of Mathematical Functions. New York: Dover, 1970.

[15] S. Zhao, H. Heer, A. A. Ioannides, M. Wagener, and H. Halling, and H. W. Müller-Gärtner. (1996). Interpolation of magnetic fields and their gradients for MEG data with 3-D spline functions [Online]. Available: citeseer.ist.psu.edu/11751.html

[16] M. Wagner, M. Fuchs, R. Drenckhahn, H. A. Wischmann, T. Köhler , and A. Theißen, "Automatic generation of BEM and FEM meshes," NeuroImage, vol. 5, no. 4, p. S389, 1997.

[17] M. Wagner, M. Fuchs, H. A. Wischmann, K. Ottenberg, and O. Dössel, "Cortex segmentation from 3-D MR images for MEG reconstructions," in Biomagnetism: Fundamental Research and Clinical Applications. Amsterdam, The Netherlands: Elsevier/IOS Press, 1995, pp. 433-438.

[18] W. H. Press, B. P. Flannery, S. A. Teukolsky, and W. T. Vetterling, Numerical Recipes. Cambridge, U.K.: Cambridge Univ. Press, 1986.

[19] D. M. Allen, "The relationship between variable selection and data augmentation and a method for prediction," Technometrics, vol. 16, no. 1, pp. 125-127, 1974.

[20] J. C. de Munck, B. van Dijk, and H. Spekreijse, "Mathematical dipoles are adequate to describe realistic generators of human brain activity," IEEE Trans. Biomed. Eng., vol. 35, no. 11, pp. 960-966, Nov. 1988.

[21] J. C. de Munck, "A linear discretization of the volume conductor boundary integral equation using analytically integrated elements," IEEE Trans. Biomed. Eng., vol. 39, no. 9, pp. 986-990, Sep. 1992.

[22] M. S. Hämäläinen and J. Sarvas, "Realistic conductivity geometry model of the human head for interpretation of neuromagnetic data," IEEE Trans. Biomed. Eng., vol. 36, no. 2, pp. 165-171, Feb. 1989.

[23] H. A. Schlitt, L. Heller, R. Aaron, E. Best, and D. M. Ranken, "Evaluation of boundary element methods for the EEG problem: Effect of linear 
interpolation," IEEE Trans. Biomed. Eng., vol. 42, no. 1, pp. 52-58, Jan. 1995.

[24] J. W. H. Meijs, O. W. Weier, M. J. Peters, and A. van Oosterom, "On the numerical accuracy of the boundary element method," IEEE Trans. Biomed. Eng., vol. BME-36, no. 10, pp. 1038-1049, Oct. 1989.

[25] H. S. Wilf, Mathematics for the Physical Sciences. New York: Dover, 1962.

[26] S. Kakutani, "Spectral analysis of stationary Gaussian processes," in Proc. 4th Berkeley Symp. Math. Stat. Probab., Univ. California, Berkeley, 1961, vol. 2, pp. 239-247.

[27] S. A. Smolyak, "Quadrature and interpolation formulas for tensor products of certain classes of functions," Sov. Math. Dokl., vol. 4, pp. 240-243, 1963.

[28] G. W. Wasilkowski and H. Wozniakowski, "Explicit cost bounds of algorithms for multivariate tensor product problems," J. Complexity, vol. 8, pp. 337-392, 1995.

[29] F. Heiss and V. Winschel. (2006, Apr.) Estimation with numerical integration on sparse grids. Department of Economics, University of Munich, Munich, Germany, Discussion Paper 2006-15 [Online]. Available: http://epub.ub.uni-muenchen.de

[30] T. F. Oostendorp, J. Delbecke, and D. F. Stegeman, "The conductivity of the human skull: Results of in vivo and in vitro measurements," IEEE Trans. Biomed. Eng., vol. 47, no. 11, pp. 1467-1492, Nov. 2000.

[31] Y. Zhang, W. van Drongelen, and B. He, "Estimation of in vivo brain-toskull conductivity ratio in humans," Appl. Phys. Lett., vol. 89, no. 22, pp. 223903-1-223903-3, 2006.

[32] K. Wendel and J. Malmivuo, "Correlation between live and post mortem skull conductivity measurements," in Proc. 28th IEEE Eng. Med. Biol. Soc. (EMBS) Annu. Int. Conf., New York, 2006, pp. 4285-4288.

[33] J. C. Mosher, M. E. Spencer, R. M. Leahy, and P. S. Lewis, "Error bounds for MEG and EEG dipole source localization," Electroencephalogr. Clin. Neurophysiol., vol. 86, pp. 303-321, 1993.

[34] B. M. Radich and K. M. Buckley, "EEG dipole localization bounds and MAP algorithms for head models with parameter uncertainties," IEEE Trans. Signal Process., vol. 42, no. 3, pp. 233-241, Mar. 1995.

[35] C. H. Muravchik and A. Nehorai, "EEG/MEG error bounds for a static dipole source with a realistic head model," IEEE Trans. Signal Process., vol. 49, no. 3, pp. 470-484, Mar. 2001.

[36] N. von Ellenrieder, C. H. Muravchik, and A. Nehorai, "Effect of head shape variations in magnetoencephalography," in New Frontiers Biomagnetism. (Congress Series 1300), Jun. 2007, pp. 181-184.

[37] N. von Ellenrieder, C. H. Muravchik, and A. Nehorai, "Effect of head shape variations on the EEG forward problem," presented at the 1st Br.Cuban Workshop Neuroimaging, Techn. Appl., Havana City, Cuba, Nov. 2006.

[38] H. M. Huizenga, J. C. de Munck, L. J. Waldorp, and R. P. P. P. Grasman, "Spatiotemporal EEG/MEG source analysis based on a parametric noise covariance model," IEEE Trans. Biomed. Eng., vol. 49, no. 6, pp. 533539, Jun. 2002.

[39] E. Huigen, A. Peper, and C. A. Grimbergen, "Investigation into the origin of the noise of surface electrodes," Med. Biol. Eng. Comput., vol. 40, pp. 332-338, 2002.

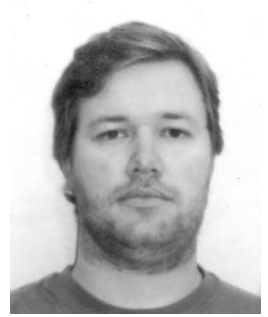

Nicolás von Ellenrieder (S'01-M'06) was born in Argentina on April 27, 1975. He recieved the Graduate degree in electronics engineering and the Ph.D. degree in electrical engineering from the National University of La Plata, Buenos Aires, Argentina, in 1998 and 2005, respectively.

He was a Visiting Researcher at Washington University in St. Louis, St. Louis, MO, in 2006. He is currently an Assistant Professor in the Department of Electrical Engineering, National University of La Plata, where he is also a member of the Industrial Electronics, Control, and Instrumentation Laboratory (LEICI). His current research interests include digital and statistical signal processing with biomedical and communication applications.

Dr. Ellenrieder is a member of the Consejo Nacional de Investigaciones Científicas y Técnicas.

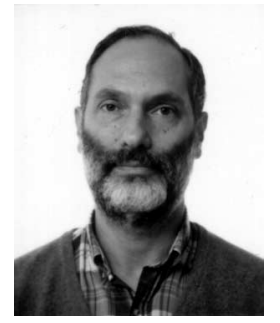

Carlos H. Muravchik (S'81-M'83-SM'98) was born in Argentina on June 11, 1951. He received the Graduate degree in electronics engineering from the National University of La Plata, Buenos Aires, Argentina, in 1973, and two M.Sc. degrees in electrical engineering and statistics and the Ph.D. degree in electrical engineering from Stanford University, Stanford, CA, in 1980, 1983, and 1983, respectively.

He was a Visiting Professor at Yale University in 1983 and 1994, at the University of Illinois at Chicago in 1996, 1997, 1999, and 2003, and at the Washington University in St. Louis, St. Louis, MO, in 2006. He is currently a Professor in the Department of Electrical Engineering, National University of La Plata, where he is also a member of the Industrial Electronics, Control, and Instrumentation Laboratory (LEICI). His current research interests include the area of statistical signal and array processing with biomedical, control, and communication applications, and nonlinear control systems

Prof. Muravchik is a member of the Comisión de Investigaciones Científicas de la Pcia de Buenos Aires. Since 1999, he is a member of the Advisory Board of the journal Latin American Applied Research and is currently an Associate Editor of the IEEE TRANSACTIONS ON SIGNAL PROCESSING.

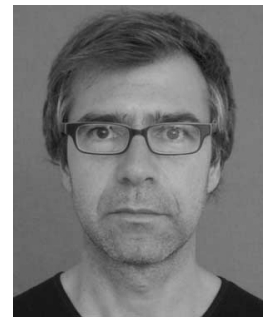

Michael Wagner received the B.A. degree in philosophy from the Philosophische Hochschule S. J., Munich, Germany, in 1989, the M.S. degree in electrical engineering from the Technical University of Munich, Munich, in 1992, and the Ph.D. degree from Hamburg Technical University, Hamburg, Germany, in 1998.

In 1992, he was with Philips Research Labs. Hamburg, where he started working on the electroand magnetoencephalography (EEG/MEG) inverse problem. Since 2000, he has been with Neuroscan (now a division of Compumedics). As Senior Scientist, his main field of work is the Curry software, where he is involved with research, software development, training, and helpdesk.

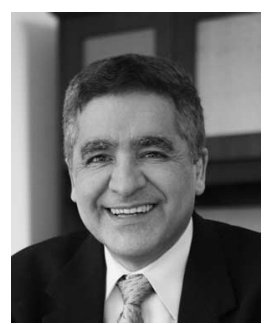

Arye Nehorai (S'80-M'83-SM'90-F'94) received the B.Sc. and M.Sc. degrees in electrical engineering from the Technion, Haifa, Israel, and the Ph.D. degree in electrical engineering from Stanford University, Stanford, CA.

From 1985 to 1995 , he was a Faculty Member in the Department of Electrical Engineering, Yale University, New Haven, CT. In 1995, he joined as a Full Professor in the Department of Electrical Engineering and Computer Science, University of Illinois, Chicago, IL, where from 2000 to 2001, he was Chair of the Electrical and Computer Engineering Division, which then became a new department. In 2001, he was named University Scholar of the University of Illinois In 2006, he became the Chairman of the Department of Electrical and Systems Engineering, Washington University in St. Louis (WUSTL), St. Louis, MO. He is the inaugural holder of the Eugene and Martha Lohman Professorship and the Director of the Center for Sensor Signal and Information Processing at WUSTL since 2006. He is the Principal Investigator of the new Multidisciplinary University Research Initiative Project entitled Adaptive Waveform Diversity for Full Spectral Dominance.

Dr. Nehorai has been a Fellow of the Royal Statistical Society since 1996. He was the Editor-in-Chief of the IEEE TRANSACTIONS ON SIGNAL PROCESSING during the years 2000-2002. From 2003 to 2005, he was Vice President (Publications) of the IEEE Signal Processing Society (SPS), Chair of the Publications Board, member of the Board of Governors, and member of the Executive Committee of this Society. From 2003 to 2006, he was the Founding Editor of the special columns on Leadership Reflections in the IEEE SIGNAL PROCESSING MAGAZINE. He was co-recipient of the IEEE SPS 1989 Senior Award for Best Paper with P. Stoica, coauthor of the 2003 Young Author Best Paper Award, and co-recipient of the 2004 Magazine Paper Award with A. Dogandzic. He was elected as a Distinguished Lecturer of the IEEE SPS for the term 2004 to 2005 and received the 2006 IEEE SPS Technical Achievement Award. 